\title{
Screening of Toxic Chemicals in a Single Drop of Human Whole Blood Using Ordered Mesoporous Carbon as a Mass Spectrometry Probe
}

\author{
Xiu Huang, ${ }^{\dagger, \dagger}$ Qian Liu, ${ }^{*},, \S, \|$ Jianjie Fu, ${ }^{\dagger}$ Zhou Nie, ${ }^{\dagger}$ Ke Gao, ${ }^{\dagger}$ and Guibin Jiang ${ }^{\dagger, \|}$ \\ ${ }^{\dagger}$ State Key Laboratory of Environmental Chemistry and Ecotoxicology, Research Center for Eco-Environmental Sciences, \\ Chinese Academy of Sciences, Beijing 100085, China \\ ${ }^{\ddagger}$ State Key Laboratory of Chemo/Biosensing and Chemometrics, College of Chemistry and Chemical Engineering, \\ Hunan University, Changsha 410082, China \\ ${ }^{\S}$ Institute of Environment and Health, Jianghan University, Wuhan 430056, China \\ "College of Resources and Environment, University of Chinese Academy of Sciences, Beijing 100190, China
}

Supporting Information

ABSTRACT: Surface-enhanced laser desorption/ionization (SELDI) is a versatile and high-throughput mass spectrometry (MS) technique that uses a probe for extraction, enrichment, desorption, and ionization of target analytes. Here we report ordered mesoporous carbon as a new SELDI probe for rapid screening and identification of trace amount of toxic chemicals in a single drop of human whole blood without complicated sample preparation procedures. We demonstrate that ordered mesoporous carbon not only can selectively enrich a wide variety of low-mass toxic compounds from whole blood samples but also can be used as an excellent matrix to assist the laser desorption/ionization process of small molecules with low background noise, high repeatability, and good salt tolerance. High sensitivity (detection limits at ppt levels) and good reproducibility for typical toxic compounds were obtained. With CMK-8 as a SELDI probe, we successfully identified and screened six perfluorinated compounds in a single drop of whole blood collected from workers in a perfluorochemical plant. The method was also validated with complex samples such as human urine and environmental water samples. With distinct advantages such as simplicity, rapidness, minimal sample requirement, and high reliability, this method keeps great promise for various aspects of application.

$\mathrm{H}$ igh-throughput mass spectrometry (MS) techniques for toxic chemicals are highly desired in many fields such as modern healthcare, public safety, forensics, exposomics, and environmental health studies. ${ }^{1-4}$ Surface-enhanced laser desorption/ ionization time-of-flight mass spectrometry (SELDI-TOF MS), a variant mode of matrix-assisted laser desorption/ionization (MALDI)-TOF MS, provides a potentially powerful tool for high-throughput screening. ${ }^{5-7}$ SELDI-TOF MS uses a probe for extraction, enrichment, desorption, and ionization of target analytes. The probe is the key of SELDI technique and an ideal probe must satisfy two requirements: (1) it is capable of selectively capturing and enriching the target compounds from complex sample matrix, and (2) it can serve as a highly efficient matrix or surface to assist the LDI process in MS detection. However, only a few materials have been reported to be available as SELDI probes ${ }^{8-13}$ due to such rigorous requirements. This seriously limits the application of the SELDI technique. Until now, SELDI-TOF MS has mainly gained applications in clinical proteomics, whereas its applications in other fields are rarely reported. Therefore, development of new probes with high sensitivity and selectivity is of great significance for the SELDI MS technique.
Ordered mesoporous carbon, with intriguing properties such as well-ordered nanopore structure, ultrahigh surface area, tunable pore size, thermal and chemical stability has shown tremendous potentials in various fields, including separation, catalyst supports, adsorption, energy storage/conversion, and biomedical engineering. ${ }^{13-17}$ In particular, the nanosized pore structure endows ordered mesoporous carbon the ability of size-selectivity in separation of different-sized biopolymers and small molecules. ${ }^{18-22}$ Its ultrahigh surface area can also provide high adsorption capacity for the enrichment. ${ }^{23}$ In combination with high optical absorptivity and thermal conductivity, ordered mesoporous carbon is expected to meet the requirements for the use as a SELDI probe. Despite that, to the best of our knowledge, the use of ordered mesoporous carbon as a MALDI matrix or SELDI probe is hitherto unreported.

Inspired by its exceptional properties mentioned above, we herein studied the use of ordered mesoporous carbon as a new SELDI probe. We show for the first time that ordered

Received: February 1, 2016

Accepted: March 17, 2016

Published: March 17, 2016 
mesoporous carbon is not only a highly efficient and selective enrichment material but also an excellent matrix for MALDI MS detection of small molecules. With CMK-8 as a SELDI probe, we could rapidly screen and identify toxic compounds at very low concentration (down to ppt level) in minimal volume of highly complex samples, e.g., even in a single drop of human whole blood. The analysis was very fast and facile without any complicated sample preparation procedures. More importantly, the as-proposed method was successfully applied to screen and identify toxic chemicals in a single drop of whole blood collected from workers in a perfluorochemical plant. We could identify more toxic compounds and obtain more accurate results by using the new method than using previously reported methods, thus demonstrating the potential of ordered mesoporous carbon as a reliable tool in high-throughput screening of toxic chemicals.

\section{EXPERIMENTAL SECTION}

Chemicals and Materials. Ordered mesoporous carbon (CMK-3 and CMK-8), ordered mesoporous silica SBA-15, and chemically converted graphene were purchased from XFNANO Materials Tech Co. (Nanjing, China). Ordered mesoporous silica MCM-41 was purchased from JC Nano Technology Co. (Nanjing, China). Bisphenol S (BPS) and tetrabromobisphenol A (TBBPA) were from TCI (Tokyo, Japan). 2,2,4,4'Tetrabromodiphenyl ether (BDE-47) was purchased from Accustandard (New Haven, CT). Pentachlorophenol (PCP) and estradiol (E2) were from Dr. Ehrenstorfer (Augsburg, Germany). Perfluorooctanesulfonate (PFOS), $\alpha$-cyano-4-hydroxycinnamic acid (CHCA), and bovine serum albumin (BSA) were bought from Sigma (St. Louis, MO). Humic acid (HA) was purchased from Alfa Aesar (Ward Hill, MA). Dichloromethane (DCM) and acetone of HPLC grade were from J. T. Baker (Phillipsburg, NJ). Ultrapure water from a Millipore Milli-Q system (Billerica, MA) was used throughout. All reagents were of analytical grade unless otherwise noted.

Characterization of Materials. TEM images were captured on a Hitachi H-7500 transmission electron microscope (Tokyo, Japan). FT-IR spectra were obtained on a JASCO FT-IR Fourier transform-infrared spectrometer (Victoria, BC, Canada). The samples were mixed and ground with $\mathrm{KBr}$ and then pressed into transparent disks for measurement. UV-visible absorption spectra were obtained on a Shimadzu UV-3600 UV-vis-NIR spectrophotometer (Kyoto, Japan). $\mathrm{X}$-ray diffraction (XRD) patterns were obtained on a PANalytical X'Pert PRO X-ray diffraction system (Almelo, The Netherlands).

MALDI- and SELDI-TOF MS. For direct MALDI-TOF MS measurement, the sample solution and the MALDI matrix dispersion was mixed at a ratio of $1: 3(\mathrm{v} / \mathrm{v})$, and $2 \mu \mathrm{L}$ of the mixture was placed onto a stainless steel MTP target frame III (Bruker Daltonics) followed by air drying.

For SELDI-TOF MS analysis (see Figure 1), the ordered mesoporous carbon probe was dispersed in water at $30 \mathrm{mg} / \mathrm{mL}$ by the aid of ultrasonication. Afterward, $400 \mu \mathrm{L}$ of the probe dispersion was added in $10 \mathrm{~mL}$ of sample solution to enrich the target analytes. The mixture was incubated in a shaking table at $25^{\circ} \mathrm{C}$ for $1 \mathrm{~h}$. The probe was then collected by centrifugation at $7000 \mathrm{rpm}$ for $15 \mathrm{~min}$, and the supernatant was discarded. Then, $3 \mathrm{~mL}$ of DCM/acetone $1: 1(\mathrm{v} / \mathrm{v})$ was added to the probe to assist the desorption of the analytes. The mixture was sonicated for $30 \mathrm{~min}$ and concentrated to $20 \mu \mathrm{L}$ under a gentle nitrogen stream. Finally, $1 \mu \mathrm{L}$ of the mixture was subjected to MALDI-TOF MS measurement.

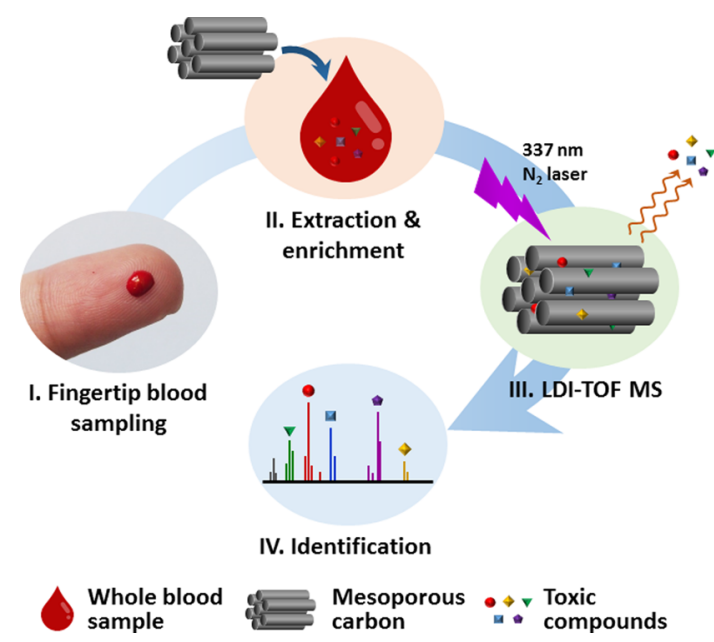

Figure 1. Scheme showing the procedures for the screening of toxic chemicals in a single drop of human whole blood.

MALDI-TOF MS was performed on a Bruker Daltonics Autoflex III Smartbean MALDI-TOF mass spectrometer in reflector mode controlled by the FlexControl software. A $337 \mathrm{~nm}$ nitrogen laser with the frequency of $100 \mathrm{~Hz}$ was used, and the laser power was set to $40 \%$. The spectra were recorded by summing 200 laser shots. Since the MS spectra obtained in negative ion mode were clearer than those in positive ion mode, ${ }^{24}$ the MS experiments were performed in negative ion mode.

Whole Blood Sample Analysis. In optimization experiments, the whole blood samples were collected from a healthy adult volunteer in Beijing, China, and stored at $-20{ }^{\circ} \mathrm{C}$. In screening experiments, the whole blood samples were collected by the fingertip blood sampling method from 25 workers in a perfluorochemical plant located in Wuhan, China, and stored at $-20{ }^{\circ} \mathrm{C}$. Prior to analysis, a single drop of whole blood sample $(\sim 20 \mu \mathrm{L})$ was diluted in $1.5 \mathrm{~mL}$ of water and then $400 \mu \mathrm{L}$ of the probe dispersion $(30 \mathrm{mg} / \mathrm{mL})$ was added. The subsequent procedures were the same as described in the SELDI-TOF MS analysis.

In addition to human whole blood samples, we also tested the methods with human urine and river water samples. Human urine samples were collected from healthy adult volunteers in Beijing, China, and stored at $-20^{\circ} \mathrm{C}$. River water samples were collected from the Xiaoling River in Beijing, China, and stored at $4{ }^{\circ} \mathrm{C}$.

\section{RESULTS AND DISCUSSION}

Characterization of Ordered Mesoporous Materials. To explore the ability of ordered mesoporous materials as SELDI probes, we studied four ordered mesoporous materials, including two mesoporous carbons (CMK-3 and CMK-8) and two mesoporous silicas (SBA-15 and MCM-41). Prior to testing their performance in MS, we characterized these materials by different techniques. TEM measurement (Figure 2A,B) indicated that these materials had well-ordered narrow pores. The average pore sizes obtained by the Brunauer-EmmettTeller (BET) method ranged from 4.7 to $6.5 \mathrm{~nm}$. All tested materials showed larger specific surface area than $500 \mathrm{~m}^{2} / \mathrm{g}$, suggesting a high adsorption capacity for the enrichment. Figure $2 \mathrm{C}$ shows absorption spectra of the ordered mesoporous materials. The ordered mesoporous carbons (CMK-3 and CMK-8) had a strong optical absorption over a wide wavelength 

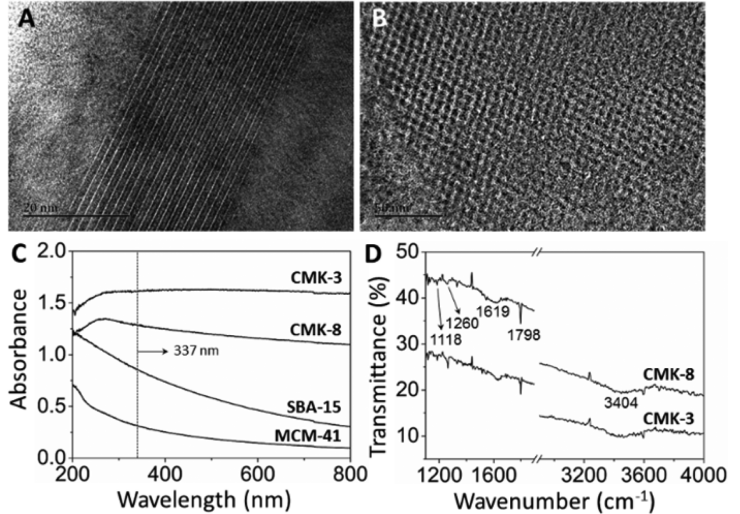

Figure 2. Characterization of ordered mesoporous materials: (A) typical TEM image of CMK-3, (B) typical TEM image of CMK-8, (C) UV-visible absorption spectra of CMK-3, CMK-8, SBA-15, and MCM-41, and (D) FT-IR spectra of CMK-3 and CMK-8.

range (including $337 \mathrm{~nm}$ that was used in MALDI) to ensure a high energy absorption capability in MALDI. In contrast, the optical absorption of ordered mesoporous silicas (SBA-15 and MCM-41) was much lower than that of ordered mesoporous carbon. Figure 2D shows the FT-IR spectra of CMK-3 and CMK-8. Both materials had a broad peak at $3404 \mathrm{~cm}^{-1}$ due to the $\mathrm{O}-\mathrm{H}$ stretching vibration. The peaks at 1798,1619 , and $1118 \mathrm{~cm}^{-1}$ were assigned to the $\mathrm{C}=\mathrm{O}$ stretching vibration, the skeletal ring vibrations, and the stretching vibration of the $\mathrm{C}-\mathrm{O}$ bond, respectively. The structures of CMK-3 and CMK- 8 were also characterized by XRD (Supporting Information Figure S1).

Use of Ordered Mesoporous Carbon as a MALDI Matrix. For the use as a SELDI probe, a prerequisite for the material is that it can be used as a MALDI matrix. As a result, we first evaluated the performance of ordered mesoporous carbon as a matrix in MALDI-TOF MS. A variety of typical toxic chemicals with molecular weight $<1000 \mathrm{Da}$ were selected as model analytes (Table 1), including brominated flame

Table 1. Feature Peaks and LODs $(\mathrm{S} / \mathrm{N}=3)$ of Typical Toxic Compounds

\begin{tabular}{llcl}
\multicolumn{1}{c}{ cmpd } & $m / z$ & LOD $(\mathrm{pg} / \mathrm{mL})^{a}$ & $\mathrm{LOD}^{\prime}(\mathrm{pg} / \mathrm{mL})^{b}$ \\
BPS & 248.3 & 10 & 62 \\
BDE-47 & 250.1 & 0.5 & 3.3 \\
PCP & 264.2 & 0.1 & 0.6 \\
E2 & 270.5 & $5.0 \times 10^{2}$ & $4.5 \times 10^{2}$ \\
PFOS & 498.5 & 1 & 6 \\
TBBPA & 542.4 & $1.0 \times 10^{2}$ & $3.3 \times 10^{2}$
\end{tabular}

${ }^{a}$ LOD: detection limit in pure water sample based on the highest feature peaks of the analyte. ${ }^{b} \mathrm{LOD}^{\prime}$ : detection limit in whole blood sample based on the highest feature peaks of the analyte.

retardants (TBBPA and BDE-47), a synthetic fluorosurfactant (PFOS), an endocrine disrupter estradiol (E2), an organochlorine pesticide (PCP), and an industrial adhesive (BPS). All these chemicals are widely used in industrial or consumer products but have been demonstrated to have potential risks to human health or ecosystems.

We compared the performance of different materials (including CMK-8, CMK-3, SBA-15, MCM-41, graphene, and a conventional organic matrix CHCA) as MALDI matrixes in detection of these typical toxic compounds. Notably, graphene, a two-dimensional carbon nanostructure, ${ }^{25-28}$ has recently been reported to be a valuable matrix material for MALDI, ${ }^{29-31}$ so it was also compared in this study. The materials were directly added to the mixed standard solutions for MALDITOF MS detection. The results are shown in Figure 3, and the

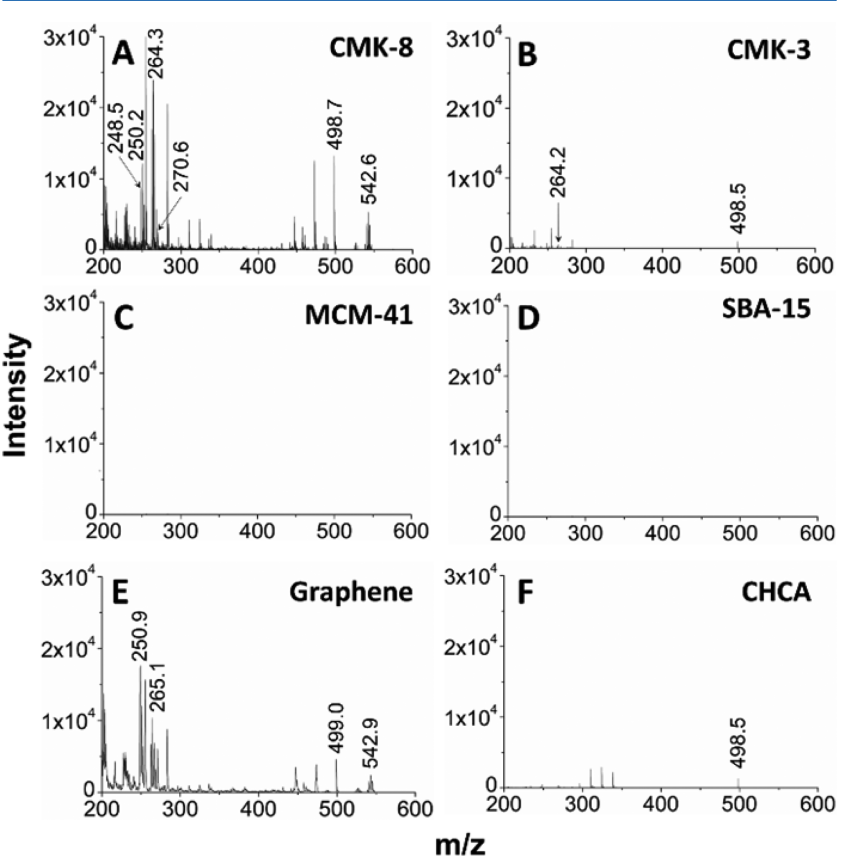

Figure 3. Comparison of performance of different materials as matrixes in MALDI-TOF MS detection of typical toxic chemicals (BPS, BDE-47, PCP, E2, PFOS, and TBBPA). (A) CMK-8, (B) CMK-3, (C) MCM-41, (D) SBA-15, (E) graphene, and (F) CHCA. Analyte concentration: BPS, $50 \mu \mathrm{g} / \mathrm{mL}$; BDE-47, $250 \mu \mathrm{g} / \mathrm{mL}$; PCP, $50 \mu \mathrm{g} / \mathrm{mL}$; E2, $250 \mu \mathrm{g} / \mathrm{mL}$; PFOS, $5 \mu \mathrm{g} / \mathrm{mL}$; TBBPA, $50 \mu \mathrm{g} / \mathrm{mL}$.

$m / z$ values of the feature peaks for the analytes are given in Table 1. From Figure 3, it is clear that CMK-8 showed the best performance among the tested materials. With CMK- 8 as a MALDI matrix (Figure 3A), all the six compounds could be readily detected with the strongest peak intensities. While with CMK-3 as a matrix (Figure 3B), although it has a stronger optical absorption than CMK-8, only peaks of $[\mathrm{M}-\mathrm{H}]^{-}$of PCP and PFOS could be detected at $\mathrm{m} / z 264.2$ and 498.5 with much lower peak intensities than those with CMK-8. The reason may be that CMK- 8 has a better electrical and thermal conductivity than CMK-3, as the unique 3D network of CMK-8 has better ion transport properties than the unidirectional 2D hexagonal systems of CMK-3. ${ }^{32-35}$ When SBA-15 and MCM-41 were used as matrixes (Figure $3 \mathrm{C}, \mathrm{D}$ ), none of the compounds could be detected, indicating that ordered mesoporous silica was not applicable to be a MALDI matrix due probably to its poor energy absorption and transfer properties (Figure 2C). ${ }^{36-39}$ In the case of graphene as a matrix (Figure $3 \mathrm{E}$ ), strong peaks of $[\mathrm{M}-\mathrm{H}]^{-}$of TBBPA, PFOS, PCP, and $\left[\mathrm{M}-\mathrm{C}_{6} \mathrm{H}_{3} \mathrm{Br}_{2}\right]^{-}$of BDE-47 at $\mathrm{m} / z$ 542.9, 499.0, 265.1, and 250.9 were obtained, but no peaks corresponding to BPS and E2 could be observed. As reported previously, ${ }^{11,30}$ graphene matrix might suffer from the aggregation that greatly compromised its performance in MALDI-TOF MS. Finally, when using CHCA as a matrix (Figure $3 \mathrm{~F}$ ), only a weak peak of $[\mathrm{M}-\mathrm{H}]^{-}$of PFOS was observed, while peaks for other compounds were absent, again indicating that conventional organic matrix was not suitable for small molecule analysis. We also compared the performance of different matrixes in 
terms of individual analytes, and results verified that CMK-8 showed the best performance among the tested materials (Figure S2).

CMK-8 as a matrix generated a good reproducibility in MALDI-TOF MS analysis. For comparison, we tested the shotto-shot $(n=20)$ and sample-to-sample relative standard deviations (RSDs; $n=15$ ) with both CMK- 8 and graphene as matrixes. The results are listed in Table 2 . We found that

Table 2. Reproducibility Test of the Method

\begin{tabular}{|c|c|c|c|c|c|c|}
\hline \multirow[b]{2}{*}{ cmpd } & \multicolumn{3}{|c|}{ shot-to-shot RSD $(n=20)^{a}$} & \multicolumn{3}{|c|}{$\begin{array}{l}\text { sample-to-sample RSD } \\
\qquad(n=15)^{b}\end{array}$} \\
\hline & $\begin{array}{l}\text { CMK-8 } \\
\text { matrix }^{c}\end{array}$ & $\begin{array}{l}\text { graphene } \\
\text { matrix }^{d}\end{array}$ & $\begin{array}{c}\text { whole } \\
\text { blood } \\
\text { sample }^{e}\end{array}$ & $\begin{array}{l}\text { CMK-8 } \\
\text { matrix }^{c}\end{array}$ & $\begin{array}{l}\text { graphene } \\
\text { matrix }^{d}\end{array}$ & $\begin{array}{l}\text { whole } \\
\text { blood } \\
\text { sample }^{e}\end{array}$ \\
\hline BPS & $11.0 \%$ & $44.4 \%$ & $12.2 \%$ & $7.0 \%$ & $57.8 \%$ & $8.1 \%$ \\
\hline BDE-47 & $8.1 \%$ & $55.2 \%$ & $8.0 \%$ & $8.5 \%$ & $49.8 \%$ & $5.7 \%$ \\
\hline РCP & $10.4 \%$ & $51.8 \%$ & $12.2 \%$ & $5.7 \%$ & $57.0 \%$ & $8.2 \%$ \\
\hline E2 & $9.7 \%$ & $48.9 \%$ & $11.5 \%$ & $7.6 \%$ & $64.0 \%$ & $6.8 \%$ \\
\hline PFOS & $6.7 \%$ & $40.7 \%$ & $12.1 \%$ & $4.0 \%$ & $54.6 \%$ & $6.4 \%$ \\
\hline TBBPA & $8.2 \%$ & $53.9 \%$ & $12.7 \%$ & $4.8 \%$ & $36.6 \%$ & $9.6 \%$ \\
\hline
\end{tabular}

${ }^{a}$ The shot-to-shot RSDs were measured based on 20 shots at different locations on the matrix. ${ }^{b}$ The sample-to-sample RSDs were measured based on 15 samples in different batches. ${ }^{c}$ The RSDs were obtained in MALDI-TOF MS analysis of mixed standards with CMK-8 as a matrix.

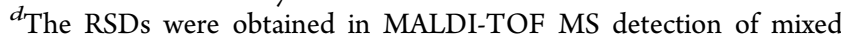
standards with graphene as a matrix. ${ }^{e}$ The RSDs were obtained in analysis of spiked whole blood samples by using CMK- 8 as a SELDI probe.

CMK-8 could form a homogeneous coating at the MALDI target to ensure reproducible analytical results. Satisfactory shot-to-shot and sample-to-sample RSDs on CMK-8 matrix were obtained in the range of $6.7-11.0 \%$ and $4.0-8.5 \%$, respectively. At the same time, due to its strongly hydrophobic nature and flexible structure, the graphene matrix was found to easily agglomerate through van der Waals interactions at the MALDI target, which caused the shot-to-shot and sample-tosample RSDs to deteriorate to $36.6-64.0 \%$. It has been reported that it is necessary to introduce some polar groups to graphene to prevent its aggregation by extra chemical modification processes, such as mild oxidation. ${ }^{30}$ Here CMK-8 showed better performance than graphene without any other chemical modifications, probably because CMK-8 inherently contained a number of polar groups such as hydroxyl and carbonyl groups (as indicated in Figure 2D), which could effectively mitigate the aggregation and favor the desorption of analytes in MALDI. Reproducibility is actually a general concern in normal MALDI-TOF MS analysis, because the cocrystallization process of analytes with conventional organic matrix would result in "hot spots". Here we show that with CMK-8 as a matrix the problem of "hot spots" could be avoided.

Considering that real samples usually contain a high content of salts, we also tested the tolerance for salt of the CMK-8 matrix. We found that the MS signals did not change significantly in the presence of $0-100 \mathrm{mM} \mathrm{NaCl}$ (Figure S3), indicating that the CMK-8 matrix was applicable for highly saline samples. Overall, the results mentioned above have demonstrated that CMK-8 is an excellent matrix for MALDITOF MS analysis of low-mass compounds.

Use of Ordered Mesoporous Carbon as a SELDI Probe. After qualification of ordered mesoporous carbon as a MALDI matrix, we further investigated its use as a SELDI probe. The probe was added to the sample to extract and enrich the target analytes, and then the probe was isolated by centrifugation and used for MS detection. It has been demonstrated that ordered mesoporous carbon is capable of selective enrichment of small molecules while excluding the interference from large molecules due to its size-selective adsorption properties. ${ }^{18,20}$ To examine whether this capability was retained in SELDI-TOF MS, we used CMK-8 as a probe to analyze spiked samples in the presence of $10 \mu \mathrm{g} / \mathrm{mL}$ BSA or HA as mimics of large molecules in biological or environmental media (Figures S4 and S5). We found that without enrichment the large molecules indeed greatly suppressed the MS signals of small molecules. This confirmed the importance of removal of large molecules in sample matrix before LDI MS analysis of small molecules. After enrichment with CMK-8, the MS signals of all compounds were fully recovered, proving that ordered mesoporous carbon was a highly selective probe for small molecules. In addition, we found that addition of a certain volume of organic solvent (i.e., DCM/acetone 1:1 (v/v)) to the probe prior to LDI MS could dramatically increase the MS responses (Figure S6). The reason may be that organic solvent could help to elute the analytes out of the nanopores of CMK-8 and thus facilitated the desorption/ionization process.

To further study the applicability of the probe for whole blood sample, we tested the method with single-drop whole blood samples spiked with typical toxic compounds. Development of high-throughput MS techniques for single-drop whole blood sample is of profound importance for rapid diagnosis and screening of health risks. Unlike serum, whole blood contains various blood cells, which would have serious specific and nonspecific adsorption for small molecules and greatly interfere with the MS detection. Thus, whole blood is regarded as a more complex matrix than serum, and direct analysis of whole blood samples is still a great challenge.

As shown in Figure 4A, direct analysis of spiked samples without using ordered mesoporous carbon as a probe, no target

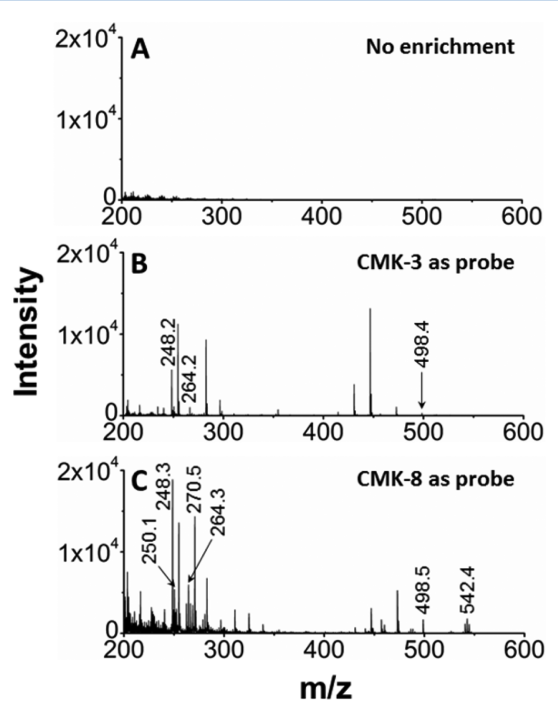

Figure 4. Comparison of CMK-3 and CMK- 8 as SELDI probe in detection of typical toxic chemicals (BPS, BDE-47, PCP, E2, PFOS, and TBBPA) in a single drop of spiked whole blood. (A) Direct analysis without enrichment, (B) using CMK-3 as probe, and (C) using CMK- 8 as probe. Spiking concentration: TBBPA, $100 \mathrm{ng} / \mathrm{mL}$; PFOS, $10 \mathrm{ng} / \mathrm{mL}$; E2, $500 \mathrm{ng} / \mathrm{mL}$; PCP, $100 \mathrm{ng} / \mathrm{mL}$; BDE-47, $500 \mathrm{ng} / \mathrm{mL}$; BPS, $100 \mathrm{ng} / \mathrm{mL}$. 
compounds could be detected. With CMK-3 as a probe, three target compounds (BPS, PCP, and PFOS) were detected at $m / z 248.2$, 264.2, and 498.4. Only using CMK-8 as a probe, all six typical compounds were detected, and the peak intensities were significantly higher than those with CMK-3. The results for unspiked whole blood samples are shown in Figure S7. Therefore, we conclude that ordered mesoporous carbon can effectively resist the interference from blood cells to small molecule analysis. Specifically, CMK-8 was more efficient than CMK-3. That may be because CMK- 8 had a wider pore size $(6.3 \mathrm{~nm})$ than CMK-3 $(4.9 \mathrm{~nm})$, and thereby it might not be easily blocked by large molecules or blood cells; furthermore, CMK-8 was a more efficient MALDI matrix than CMK-3 (see Figure 3). Therefore, CMK-8 was used as a SELDI probe in the following experiments.

Although our target sample media was human whole blood, we also validated the method with other types of complex samples, such as human urine (Figure S8) and river water samples (Figures S9). Results show that the CMK-8 probe could keep its high sensitivity and selectivity in various types of complex sample media. Some graphite-based materials such as pencil leads ${ }^{40-42}$ and modified graphene materials ${ }^{11,30}$ have also been reported as MALDI matrixes or SELDI probes. Compared with these materials, the unique size-selective adsorption properties of ordered mesoporous carbon make it more effective in resisting interference from sample matrix, thus generating a stronger ability for direct analysis of complex samples.

Analytical Performance. Table 1 lists the limits of detection (LODs; at signal-to-noise ratio $(\mathrm{S} / \mathrm{N})=3$ ) of the CMK-8 probe for typical toxic compounds in pure water and whole blood samples. It can be seen that the LODs for all the compounds were at part per trillion (ppt) or even subppt levels $(0.1-500 \mathrm{pg} / \mathrm{mL})$. Such a high sensitivity allowed the screening to be performed with a very small amount of sample, i.e., even a single drop of whole blood. Thus, the method can be applied to the direct analysis of fingertip blood samples without any other preconcentration procedures. A detailed comparison with other methods in terms of analytical sensitivity is given in Supporting Information Table S1. For most of the compounds (except for PFOS), the sensitivity of the present method was better than or comparable to that of conventional chromatography-mass spectrometry methods. The calibration curves for the typical toxic compounds are given in Table S2. The method also showed a good reproducibility in whole blood analysis. As shown in Table 2 , the shot-to-shot $(n=20)$ and sample-tosample RSDs $(n=15)$ were in the range of $8.0 \%-12.7 \%$ and $5.7 \%-9.6 \%$, respectively.

In addition to high sensitivity and reproducibility, the method also presents other distinct advantages. Because of the simple procedures and high-throughput nature of SELDI-TOF MS, it may be able to finish the analysis of hundreds of samples within several hours. Thus, this method is much faster than conventional methods that usually involve laborious and timeconsuming sample preparation steps. ${ }^{43-45}$ Furthermore, unmodified ordered mesoporous carbon plays multiple roles in the analysis and thereby eliminates the use of other materials, making the method more facile than previously reported methods. $^{18,35}$

Screening of Toxic Chemicals in Worker Whole Blood Samples. We applied the as-proposed method in identification and screening of toxic chemicals in whole blood samples collected from workers in a perfluorochemical plant. Perfluorinated compounds can persist for a long period of time in the environment and have bioaccumulation properties and potential liver toxicity. ${ }^{46-49}$ We collected fingertip whole blood samples from 25 workers from different departments. For control experiments, we also collected and analyzed samples from 5 healthy adults (nonworker). As shown in Figure 5A, none of toxic compounds could be identified in
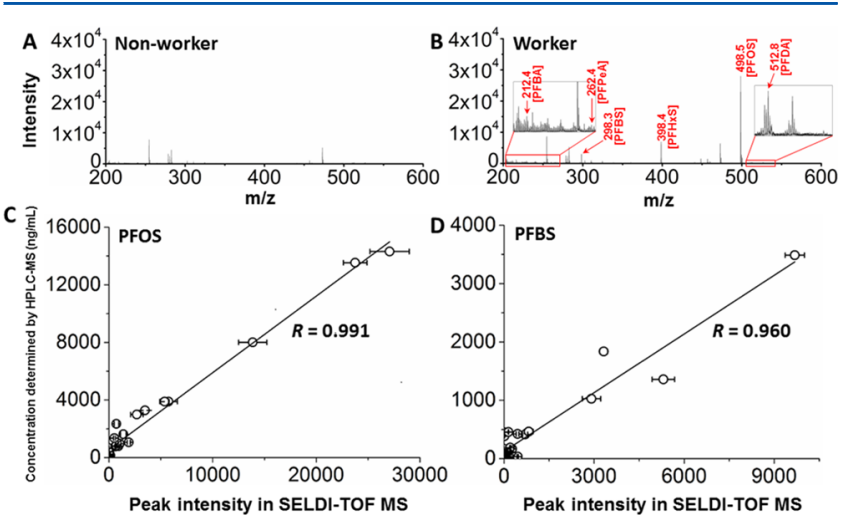

Figure 5. Rapid screening of toxic chemicals in a single drop of human whole blood from 25 workers in a perfluorochemical plant and 5 nonworkers. (A, B) Typical mass spectra of whole blood samples from nonworkers and workers and (C, D) comparison of analytical results of PFOS and PFBS obtained by SELDI-TOF MS with concentrations determined by HPLC-ESI-MS/MS.

healthy adult whole blood samples. However, in worker whole blood samples (Figure 5B), we identified six perfluorochemicals at $m / z 212.4,262.4,298.3,398.4,498.5$, and 512.8, which corresponded to the peaks of $[\mathrm{M}-\mathrm{H}]^{-}$of perfluorobutyric acid (PFBA), perfluorovaleric acid (PFPeA), perfluorobutanesulfonic acid (PFBS), perfluorohexanesulfonate (PFHxS), perfluorooctanesulfonate (PFOS), and perfluorodecanoic acid (PFDA), respectively. The detailed results are given in Table S3. Taking PFOS as an example, the peak intensity could reach $3 \times 10^{4}$, indicating high levels of perfluorochemicals in the worker blood. Notably, we herein identified more toxic compounds than in a previous work, ${ }^{18}$ in which graphene was used as a MALDI matrix. The reason may be that the CMK-8-based SELDI MS method did not involve the steps of elution and transferring of analytes between different materials, thus preventing possible loss of trace amount of target compounds. Accordingly, the result obtained here indicates that the CMK-8 is a highly efficient probe for identification of unknown toxic chemicals in complex samples.

We verified our results obtained in SELDI-TOF MS by analyzing the same set of samples using a conventional method, high-performance liquid chromatography-electrospray ionization-tandem MS (HPLC-ESI-MS/MS; see Supporting Information for experimental details). The concentrations of the identified perfluorochemicals in whole blood samples were determined by HPLC-ESI-MS/MS and given in Table S4. As shown in Figure 5C,D, the peak intensities of perfluorochemicals in SELDI-TOF MS showed excellent linear correlations $(R=0.991$ and 0.960$)$ with the concentrations obtained by HPLC-ESI-MS/MS. Data for more perfluorochemicals are given in Figure $\operatorname{S10}(R>0.932)$. The good consistency between these two techniques definitely proved the high accuracy of the CMK-8-based SELDI-TOF MS method. Particularly, the correlation was positively related with the peak intensity of the compounds. For instance, PFOS showed 
the highest peak intensity among the identified compounds, and its correlation coefficient $R$ was highly approximated to 1 . This accuracy was better than that obtained in a previous work. $^{18}$ Therefore, the CMK-8 probe not only provides a reliable tool for rapid screening of toxic compounds but also can be used for their semiquantitative or even quantitative analysis (especially for compounds with high MS responses). Finally, we should stress that the SELDI-TOF MS method was much faster and more facile than the conventional HPLCESI-MS/MS method, because the latter could not directly analyze whole blood samples and required complicated sample preparation procedures.

\section{CONCLUSIONS}

In summary, we have demonstrated that ordered mesoporous carbon can be used as a new MALDI matrix and SELDI probe. As a MALDI matrix, ordered mesoporous carbon produced high sensitivity, low background noise in the low-mass region, good reproducibility, and high tolerance for salt. As a SELDI probe, it can be used to rapidly and sensitively detect trace amount of low-mass compounds in highly complex samples (e.g., biological and environmental samples) at high throughput. We have successfully applied this probe to screen and identify toxic compounds in a single drop of human whole blood and verified the results by the conventional method. Therefore, with prominent advantages such as simplicity, rapidness, high sensitivity, minimal sample requirement, and high reliability, we believe that the present method not only provides a powerful tool for high-throughput screening of toxic chemicals but also keeps tremendous application potentials in a wide variety of fields.

\section{ASSOCIATED CONTENT}

\section{(5) Supporting Information}

The Supporting Information is available free of charge on the ACS Publications website at DOI: 10.1021/acs.analchem.6b00444.

Experimental details, supporting tables, and supporting figures (PDF)

\section{AUTHOR INFORMATION}

\section{Corresponding Author}

*E-mail: qianliu@rcees.ac.cn.

Notes

The authors declare no competing financial interest.

\section{ACKNOWLEDGMENTS}

The authors acknowledge financial support from the Chinese Academy of Sciences (Grant XDB14010400), the National Basic Research Program of China (Grants 2015CB931903 and 2015CB932003), and the National Natural Science Foundation of China (Grants 21377141, 21422509, and 91543104). Q. Liu also acknowledges the support from the Youth Innovation Promotion Association of CAS.

\section{REFERENCES}

(1) Horswell, J.; Dickson, S. J. J. Anal. Toxicol. 2003, 27, 372-376.

(2) Goldman, L. R.; Koduru, S. Environ. Health Perspect. 2000, 108, 443.

(3) Kaptein, A. A.; Helder, D. I.; Kleijn, W. C. J. Psychosom. Res. 2005, 58, 453-457.

(4) Rappaport, S. M. J. Exposure Sci. Environ. Epidemiol. 2011, 21, 5-9.
(5) Merchant, M.; Weinberger, S. R. Electrophoresis 2000, 21, 11641177.

(6) Caffrey, R. E. In The Urinary Proteome: Methods and Protocols; Humana Press: Totowa, NJ, 2010; pp 167-183.

(7) Grizzle, W. E.; Semmes, O. J.; Bigbee, W.; Zhu, L.; Malik, G.; Oelschlager, D. K.; Manne, B.; Manneaet, U. Cancer Inform. 2005, 1, 86-97.

(8) Tang, L. A. L.; Wang, J.; Loh, K. P. J. Am. Chem. Soc. 2010, 132, 10976-10977.

(9) Favre-Kontula, L.; Sattonnet-Roche, P.; Magnenat, E. Proteomics 2008, 8, 378-388.

(10) Gulbakan, B.; Yasun, E.; Shukoor, M. I.; Zhu, Z.; You, M. X.; Tan, X. H.; Sanchez, H.; Powell, D. H.; Dai, H. J.; Tan, W. H. J. Am. Chem. Soc. 2010, 132, 17408-17410.

(11) Liu, Q.; Cheng, M.; Wang, J.; Jiang, G. Chem. - Eur. J. 2015, 21, 5594-5599.

(12) Kawasaki, H.; Nakai, K.; Arakawa, R.; Athanassiou, E. K.; Grass, R. N.; Stark, W. J. Anal. Chem. 2012, 84, 9268-9275.

(13) Tang, N.; Tornatore, P.; Weinberger, S. R. Mass Spectrom. Rev. 2004, 23, 34-44.

(14) Muthu, M.; Vimala, A.; Mendoza, O. H. TrAC, Trends Anal. Chem. 2016, 76, 95-101.

(15) Ying, J. Y.; Mehnert, C. P.; Wong, M. S. Angew. Chem., Int. Ed. 1999, 38, 56-77.

(16) Davis, M. E. Nature 2002, 417, 813-821.

(17) Eddaoudi, M.; Kim, J.; Rosi, N.; Vodak, D.; Wachter, J.; O’Keeffe, M.; Yaghi, O. M. Science 2002, 295, 469-472.

(18) Wang, J.; Liu, Q.; Gao, Y.; Wang, Y.; Guo, L.; Jiang, G. Anal. Chem. 2015, 87, 6931-6936.

(19) Zhao, L.; Qin, H.; Wu, R.; Zou, H. J. Chromatogr. A 2012, 1228, 193-204.

(20) Qin, H.; Gao, P.; Wang, F.; Zhao, L.; Zhu, J.; Wang, A.; Zhang, T.; Wu, R. A.; Zou, H. Angew. Chem., Int. Ed. 2011, 50, 12218-12221.

(21) Sun, N.; Deng, C.; Li, Y.; Zhang, X. Anal. Chem. 2014, 86, 2246-2250.

(22) Teng, W.; Wu, Z.; Feng, D.; Fan, J.; Wang, J.; Wei, H.; Song, M.; Zhao, D. Environ. Sci. Technol. 2013, 47, 8633-8641.

(23) Sun, Z.; Deng, Y.; Wei, J.; Gu, D.; Tu, B.; Zhao, D. Chem. Mater. 2011, 23, 2176-2184.

(24) Lu, M.; Lai, Y.; Chen, G.; Cai, Z. Anal. Chem. 2011, 83, 31613169.

(25) Novoselov, K. S.; Fal'ko, V.; Colombo, L.; Gellert, P.; Schwab, M.; Kim, K. Nature 2012, 490, 192-200.

(26) Bitounis, D.; Ali-Boucetta, H.; Hong, B. H.; Min, D. H.; Kostarelos, K. Adv. Mater. 2013, 25, 2258-2268.

(27) Biro, L. P.; Nemes-Incze, P.; Lambin, P. Nanoscale 2012, 4, 1824-1839.

(28) Guo, S.; Dong, S. Chem. Soc. Rev. 2011, 40, 2644-2672.

(29) Dong, X.; Cheng, J.; Li, J.; Wang, Y. Anal. Chem. 2010, 82, $6208-6214$

(30) Liu, Q.; Cheng, M.; Jiang, G. Chem. - Eur. J. 2013, 19, 55615565.

(31) Kawase, T.; Yoshitake, H. Microporous Mesoporous Mater. 2012, $155,99-105$.

(32) Lang, J. W.; Yan, X. B.; Yuan, X. Y.; Yang, J.; Xue, Q. J. J. Power Sources 2011, 196, 10472-10478.

(33) Lang, J. W.; Yan, X. B.; Liu, W. W.; Wang, R. T.; Xue, Q. J. J. Power Sources 2012, 204, 220-229.

(34) Maiyalagan, T.; Alaje, T. O.; Scott, K. J. Phys. Chem. C 2012, $116,2630-2638$.

(35) Min, Q.; Zhang, X.; Chen, X.; Li, S.; Zhu, J. J. Anal. Chem. 2014, $86,9122-9130$.

(36) Baskaran, S.; Liu, J.; Domansky, K.; Kohler, N.; Li, X.; Coyle, C.; Fryxell, G.; Thevuthasan, S.; Williford, R. Adv. Mater. 2000, 12, 291294.

(37) Yang, P.; Zhao, D.; Margolese, D. I.; Chmelka, B. F.; Stucky, G. D. Nature 1998, 396, 152-155.

(38) Yang, P.; Zhao, D.; Margolese, D. I.; Chmelka, B. F.; Stucky, G. D. Chem. Mater. 1999, 11, 2813-2826. 
(39) Fan, H.; Bentley, H. R.; Kathan, K. R.; Clem, P.; Lu, Y.; Brinker, C. J. J. Non-Cryst. Solids 2001, 285, 79-83.

(40) Black, C.; Poile, C.; Langley, J.; Herniman, J. Rapid Commun. Mass Spectrom. 2006, 20, 1053-1060.

(41) Langley, G. J.; Herniman, J. M.; Townell, M. S. Rapid Commun. Mass Spectrom. 2007, 21, 180-190.

(42) Berger-Nicoletti, E.; Wurm, F.; Kilbinger, A. F.; Frey, H. Macromolecules 2007, 40, 746-751.

(43) Richardson, S. D. Anal. Chem. 2012, 84, 747-778.

(44) Jamt, R. E. G.; Gjelstad, A.; Eibak, L. E. E.; Øiestad, E. L.; Christophersen, A. S.; Rasmussen, K. E.; Pedersen-Bjergaard, S. J. Chromatogr. A 2012, 1232, 27-36.

(45) Minakata, K.; Yamagishi, I.; Nozawa, H.; Hasegawa, K.; Wurita, A.; Gonmori, K.; Suzuki, M.; Watanabe, K.; Suzuki, O. Forensic Toxicol. 2014, 32, 169-175.

(46) Kudo, N.; Kawashima, Y. Toxicol. Appl. Pharmacol. 1997, 145, 285-293.

(47) Jeon, J.; Kannan, K.; Lim, H. K.; Moon, H. B.; Ra, J. S.; Kim, S. D. Environ. Sci. Technol. 2010, 44, 2695-2701.

(48) Seacat, A.; Thomford, P.; Hansen, K.; Olsen, G.; Case, M.; Butenhoff, J. Toxicol. Sci. 2002, 68, 249-264.

(49) Yang, Q.; Abedi-Valugerdi, M.; Xie, Y.; Zhao, X. Y.; Möller, G.; Nelson, B. D.; DePierre, J. W. Int. Immunopharmacol. 2002, 2, 389397. 